\title{
Alex Demirovic
}

\section{Intellektuelle und Gesellschaftskritik heute}

Gesellschaftskritik ist eine normale Erscheinung moderner, kapitalistischer Gesellschaften, gewissermaßen eine Institution. Es ist deswegen eine abstrakte Überlegung, daß sich das soziale und intellektuelle Feld in einer Weise transformieren könnte, daß der Begriff der Kritik jede soziale Verpflichtung verlieren und ein sozialer Konsens darüber entstehen könnte, jede Form von Kritik zu unterlassen. Allerdings ist der Begriff der Gesellschaftskritik geschichtlich und vom Stand gesellschaftlicher Auseinandersetzungen bestimmt. Kritik ist in den ProzeB gesellschaftlicher Krisen immer schon involviert und kann mithin Krisen nicht nur beschleunigen und verschärfen, sondern auch selbst in eine Krise geraten. Die Geschichte linker Sozialkritik ist periodisch von solchen Prozessen der Demoralisierung gekennzeichnet. Eindrucksvoll hat eine solche Situation der exilierte Horkheimer festgehalten: »Selbst fortgeschrittene Teile der Gesellschaft werden entmutigt, von der allgemeinen Ratlosigkeit ergriffen. Auch die Wahrheit ist in ihrem Bestand an Konstellationen der Realität geknüpft. (Horkheimer 1988, 211)

Die Klage über die Depotenzierung der Wahrheit der Begriffe und die Demoralisierung der Kritik kann unter sehr spezifischen Bedingungen der Ausgangspunkt einer Kritik an der gesellschaftlichen Entwicklung sein. Doch wird diese Klage eine hilflose und möglicherweise sogar eine lächerliche Kritikstrategie, wenn die Figur des kritischen Intellektuellen selbst zum Gegenstand einer Kritik geworden ist, die sein Verhalten, seinen Willen zum Wissen, seinen Anspruch auf Wahrheit, seine Rede im Namen der Allgemeinheit, seine Sorge um die Zukunft darauf hin befragt, wieweit er wirklich das Ziel der Emanzipation vor Augen hat und ob seine Kritik nicht einer Bevormundung derer, die selbst nicht sprechen, das Wort redet. Um dieses Argument ist Horkheimers theoretische Einsicht, daß die gesellschaftskritische Theorie in den gesellschaftlichen Prozeß einbezogen sei, heute zu erweitern. Gesellschaftskritik und die mit ihr verbundenen Formen von Intellektualität haben einen Zeitkern und können folglich auch in Krisen geraten. Krise bedeutet, daß tradierte kritische Begriffe keinen selbstevidenten Hintergrund mehr zur Beurteilung einer gesellschaftlichen Situation darstellen. Kritik, die gestern noch auf selbstverständliche $\mathrm{Zu}-$ 
stimmung rechnen konnte, wird heute als dogmatisch, besserwisserisch, arrogant, lächerlich und anachronistisch zurückgewiesen. Wird dies allein als Verrat der Intellektuellen betrachtet, werden die rationellen Aspekte dieser Krise der Kritik verkannt. Die sachlich gerechtfertigten Motive des Auflösungs- und Abwanderungsprozesses aus einer Kritikformation werden nicht ernst genommen, obwohl sie doch Anlaß zur selbstkritischen Transformation von Kritikstandards sein sollten. Der Versuch, die Abwandernden durch moralischen Druck zu binden, bewirkt schließlich das Gegenteil. Denn ein solcher Kritikstandard wird nicht nur als repressiv empfunden, sondern zudem auch als schwach, weil er sich eben nicht mehr allein auf die Überzeugungskraft seiner Wahrheit verlassen kann. Die Schwäche derer, die auf bloß heroische Weise überkommene Kriterien hochhalten, läßt sich selbst wiederum in immer neuen Zugeständnissen und Nachbesserungen beobachten, mit denen sie Begriffe und Theoreme nachträglich akzeptieren, die zunächst energisch kritisiert und verworfen wurden. »Renegaten«, »Verräter « und »Abweichler«, ihre Theorien und Maßstäbe, können so schließlich in einer weiteren historischen Phase zu außerordentlicher Bedeutung gelangen, weil ihre frühere Abwanderung eben doch auch rationale Aspekte hatte. Für die historischen Verlaufsformen von Kritik, Abwanderung, Transformation der Kritikmuster und Neuformierung von gesellschaftskritischen Intellektuellen gibt es bislang, so mein Eindruck, weder historische Analysen noch eine Theoriebildung. Dies ist selbst wiederum dem Objektivismus linker Analysen geschuldet, die sich selten als ein Teil der Gesamtbewegung der von ihr kritisierten Gesellschaften verstehen - und insofern zu wenig berücksichtigen, wie sehr sie selbst ständigen Transformationen unterworfen sind oder an diesen arbeiten.

Ich will im vorliegenden Artikel weder zu einer solchen Sozialgeschichte noch zur Theorie dieses Transformismus der Linken beitragen. Weit unterhalb eines solchen Anspruchs, geht es mir im folgenden darum, anhand eines knappen Überblicks über eine Reihe von meist für den Tag bestimmten, feuilletonistischen Äußerungen zur aktuellen Rolle von Intellektuellen und des Links-Rechts-Binarismus nachzuzeichnen, welchen Herausforderungen und Einwänden sich ein zeitgemäßer Kritikbegriff stellen muß. Ein solcher Begriff soll im weiteren in Auseinandersetzung mit Michel Foucault und Michael Walzer, und nicht zuletzt im Rückgriff auf den Kritikbegriff der älteren Kritischen Theorie wenigstens angedeutet werden.

$\mathbb{1}$.

Vor wenigen Jahre erst wurde behauptet, in Deutschland habe sich die professionelle Rolle des wahrheitsorientierten, kritischen Intellektuellen eta- 
bliert (vgl. Brunkhorst 1987). Inmitten der Massendemokratie und der öffentlichen Meinung als deren sittlicher Substanz habe der Intellektuelle an der exzentrischen Peripherie seinen Platz eingenommen und übernehme dort, so Brunkhorst, die Rolle der praktischen »Vermittlung universeller Vernunftansprüche mit dem vielstimmig artikulierten Willen und den widerstreitenden Bedürfnissen der Vielen« (ebd., 41). Demgegenüber könnte das Erscheinen einer Sammlung von Aufsätzen des gehobenen konservativen Feuilletons unter dem fragenden Titel Intellektuellendämmerung? (Meyer (Hg.) 1992), in dem die Meinung vorherrscht, die Zeit der Intellektuellen sei passé, als Kampfansage konservativer Gegenintellektueller bewertet werden. Daß die Konservativen allerdings dem Zeitgeist hinterherhinken, macht Michael Krügers satirische Kriegsberichterstattung über die aktuellen Intellektuellenkämpfe deutlich. Schwerpunkte der kriegerischen Auseinandersetzungen - so seine Anspielung auf den linken Postmodernen Lyotard, der den Intelletuellen schon in den 80er Jahren ein Grabmal setzte (vgl. Lyotard 1985) - seien »eigentümlicherweise die Friedhöfe geworden, auf denen die 'Grablegung des Intellektuellen' zelebriert wird « (Krüger, FR vom 21.1.1993). Der Intellektuelle erscheint als schon längst obsolete Figur, weil die Gewißheiten dahin zu sein scheinen, auf die sich seine Kritik an der eigenen Gesellschaft berief. Gerade weil er im Begriff sei, die Bühne der deutschen Nachkriegsgeschichte zu verlassen, könne er, wie Micha Brumlik mit einem Hegelischen Argument behauptet, zum Gegenstand der Reflexion werden. $\gg$ Die Geschichte der bundesdeutschen Intellektuellen kann bald geschrieben werden, denn sie ist beinahe vorbei« (Brumlik, taz vom 8.9.1992).

Hegelianer könnten aus dieser nekrophilen Selbstreflexion neue Hoffnung schöpfen, denn vermutlich würden es ja Intellektuelle sein, die die Geschichte der Intellektuellen schreiben. Dialektik würde den Intellektuellen durch Reflexion auf seine Obsoleszenz in neuer Gestalt wiedererstehen lassen. Allerdings erfreut sich die Beschäftigung der Intellektuellen mit sich selbst seit jeher keiner besonders großen Beliebtheit. Der Hinweis auf bloße Selbstbeschäftigung ist seinerseits ein Topos zeitdiagnostischer Gesellschaftskritik. Es wird als ein Zeichen der Malaise, der Kopflahmheit, des Mangels an Ideen und Phantasien verstanden, daß die »Hauptsorge der Intellektuellen der Funktion der Intellektuellen gilt « (Michael Krïger) schreibt ein Intellektueller über Intellektuelle und bestätigt prompt, daß sich Intellektuelle trotz aller Appelle aus der Selbstreferenz nicht herausbewegen können oder anders, daß sie mit ihren Äußerungen immer intellektuelle Verhältnisse eingehen müssen.

Die Rede des Intellektuellen über sein historisches Entschwinden wird als der Versuch verdächtigt, seine Existenz auf immer neue Weise selbstrefe- 
rentiell über sein Ende hinaus zu verlängern. »Als Intellektueller gilt, wer imstande ist, die Frage nach dem Intellektuellen zu formulieren: analog zur Hand M.C. Eschers, die sich zeichnet, wie sie die Hand M.C. Eschers zeichnet, wie sie ... ad infinitum« (Macho 1992, 50). Der hegelianisierenden Erwartung einer neuen Morgenröte der Intellektuellen versucht auch die Systemtheorie vorzubeugen, die die Selbstbezüglichkeit des intellektuellen Feldes kritisiert, obwohl doch von ihr dafür am ehesten noch theoretisches Verständnis hätte erwartet werden können. Luhmann versucht, eine Antwort darauf zu geben, warum es zu diesem Autismus der intellektuellen Diskussion kommt. »'Postmodern' ist ... die Formel für Intellektuelle, die den Glauben an die von ihnen bevorzugten Theorien verloren haben, aber trotzdem beisammenbleiben und darüber reden möchten« (Luhmann 1992, 65). Mit diesem Argument weist er auf einen Aspekt hin, der auch Gramsci in seinen Überlegungen zur Funktion der Zivilgesellschaft und der Rolle der Intellektuellen schon beschäftigt hat. Eine Krise der Theorie ist ein soziales Verhältnis, d.h. das Verhältnis der Intellektuellen zur Theorie variiert: die Theorie und die mit ihr verbundenen Kriterien sind nicht mehr der Sinnkosmos, in dem die Intellektuellen als im Wahren leben. Die Krise im intellektuellen Feld erzeugen die Intellektuellen durch ihre Rede übereinander, indem sie die Maßstäbe ihrer Rede in ein neues Licht setzen und sich damit in einem molekularen Proze $ß$ zu neuen Reaktionen, zu einem neuen Verhältnis zur Theorie und zur Wahrheit zwingen. In einer solchen Übergangsphase, die als unübersichtlich, ambivalent, riskant und unsicher erlebt wird, schieben sich Probleme der moralischen Begründung in den Vordergrund.

Wie es zu den gegenwärtig beobachteten Veränderungen des Verhältnisses gesellschaftskritischer, linker Intellektueller zu ihren Theorien und zur angeblich autistischen Selbstbeschäftigung kommt, ist selbst ein Gegenstand zahlreicher Ausführungen zur Bestimmung von Intellektualität. Einer gewissen Beliebtheit erfreut sich die Erklärung dieses Sachverhalts durch die einschneidenden historischen Veränderungen seit 1989. Die maßgebende Strömung von Intellektuellen in der Bundesrepublik sei so sehr auf die »Möglichkeit einer (wie immer) zu denkenden sozialistischen Alternative sowie auf die Konstellation vielfältiger Verbesserungen der Lebenshaltung im Nachkriegseuropa bezogen, daß sie sich auf die Realität seit 1989 kaum wird einstellen können « (Meier 1992, 85). Der linke Glaube sei eher an Besitzstandswahrung als an der Freiheit der Menschen in Osteuropa orientiert. Zukunft werde für sie zur Bedrohung. Insofern sei die Linke weltfremd geworden und füge sich gänzlich in die lange Tradition deutscher Weltfremdheit ein (vgl. Ritter 1993, 110f.). 
Die Bemerkung Christian Meiers bekräftigt indirekt noch einmal die von vielen Intellektuellen vertretene Ansicht, daß Intellektuelle vor allem links und sozialistisch sind, Konservative hingegen anti-intellektuell; und sie verweist darauf, wie eng Äußerungen über Intellektuelle mit dem LinksRechts-Binarismus zusammenhängen. Die Konsequenz dieser Verknüpfung ist, so wird nahegelegt, daß es mit der Auflösung des Sozialismus keine Linken und folglich auch keine Intellektuellen mehr geben wird. Damit wird eine weitere Selbstreferenz des intellektuellen Feldes virulent: ohne Linke gibt es auch keine Rechte, ohne linke Intellektuelle keine rechte Anti-Intellektualität. Da die Rechte aber ohne ihr Gegenteil nicht existieren könne, gebe es Bemühungen, dieses zu revitalisieren. Im Sinne einer Feindschaft, die ihr Gegenüber notwendig zur Selbstkontinuierung brauche, suche sie die linken Intellektuellen »durch regelmäßige Attacken wieder zum Leben zu erwecken« oder sich »zusammenzureimen« (Greiner, Die Zeit vom 18.9.1992). Diesen Versuchen billigt Greiner allerdings wenig Aussicht auf Erfolg zu. Er geht insofern weiter als Meier, als die Ursache für die Auflösung des linksintellektuellen Lagers nämlich nicht das Scheitern des Realsozialismus sei, der ja nie das Identifikationsobjekt der »(wahren) Linken « gewesen sein soll. Vielmehr sei mit dem Sozialismus etwas viel Grundlegenderes gescheitert. Die Utopie nämlich, daß sich die mit der praktischen Verwirklichung der universalistischen Normen von Gleichheit und Freiheit zwangsläufig einstellenden Widersprüche in irgendeiner Zukunft aufheben lassen könnten. Zwar wollten alle Gleichheit, Freiheit, Würde und Gerechtigkeit. Doch die Probleme würden beginnen, »wenn man begreift, daß die totale Verwirklichung dieser Ideale dem Versuch ähnelt, mehrere Kamele gleichzeitig durch ein Nadelöhr zu zwingen« (Greiner). Die historische Situation sei durch das Ende der Utopie - der kapitalistischen wie der sozialistischen - bestimmt (Lepenies 1992, 23). Nachdem dies ersichtlich wurde, so beobachtet auch Henning Ritter, liefen den Intellektuellen nun die Gläubigen fort, und die Intellektuellen selbst würden ratlos. Mit der Selbstaufhebung der Geschichtsphilosophie sei schließlich auch die Geschichte der Intellektuellen an ihr Ende gekommen (vgl. Ritter 1992, 251). Und mit den Intellektuellen auch der Links-RechtsBinarismus, der aufs engste mit dem Projekt der Aufklärung und eines geschichtsphilosophisch determinierten Fortschritts verbunden gewesen sei. Diese feuilletonistischen Einschätzungen der Lage der Intellektuellen und der linken Gesellschaftskritik werden ihrerseits kritisiert. So erklärt Wolfgang Pohrt ideologiekritisch die Veränderungen im intellektuellen Feld als Ergebnis von Konformismus und Verrat. Viele Intellektuellen seien früher aus Konformismus Marxisten gewesen, als noch nicht eindeutig feststand, ob das westliche oder östliche System siegen würde. Insbesondere der li- 
bertäre, westliche Marxismus sei eine wunderbare Konstruktion gewesen, um die abwartende Haltung zu rechtfertigen. Dies erklärt die Beobachtung, daß doch eigentlich jetzt, nach dem Ende der autoritären realsozialistischen Regimes, der Zeitpunkt des emanzipatorischen Marxismus hätte kommen müssen.

»Die verabscheuten Machthaber im Osten sind weg. Schön für den besseren Marxismus, sollte man denken. Jetzt kann er richtig loslegen und seine ganze Energie auf die Kritik des Kapitals konzentrieren. Aber keine Spur davon. Mit den schlechten Marxisten sind auch die guten verschwunden.« (Pohrt 1993, 26) Obwohl niemand mehr Sehnsucht nach der revolutionären Umwälzung der kapitalistischen Gesellschaft zeige, forderten »lauter mustergültig Resozialisierte einander reihum dazu auf, von den verhängnisvollen alten Irrtümern abzulassen. .. Es reicht den vormals Linksradikalen nicht, daß sie mit dem Alter wurden, was sie sind; sie wollen kraft Einsicht und freiem Entschluß geworden oder noch besser schon immer gewesen sein, was gesellschaftlicher Druck und eigene Nachgiebigkeit aus ihnen machten. Gemeinsam mit dem früheren Gegner feiern sie ihre eigene politische und moralische Niederlage als strahlenden Sieg der Vernunft« (ebd.).

In seiner Ideologiekritik beobachtet Pohrt richtig auch einen neuen intellektuellen Stil, eine merkwürdige Form des intellektuellen Heroismus. Simultan würden zahllose Autisten für sich in Anspruch nehmen, einsame Geister mit dem Mut zur eigenen Meinung zu sein. Sicherlich ist dieser Anspruch auf Nonkonformismus auch eine leere Geste, die kritisiert werden muß: die Tradition der radikalen Kritik wird formell fortgesetzt, indem fiktiven Gegnern Begriffe einer Gesellschaftskritik unterstellt werden, die vielleicht nur die Kritiker früher einmal vertreten haben. Sie gelten als Meinungsmacher, weil sie sich als Linke mit lautstark vorgebrachten Provokationen über das Kollektivsubjekt »die Linke« äußern. Indem sie diese kommentieren und sich kritisch distanzieren, konstruieren sie sie allerest ebenso wie die Legitimität, sich selbst als Linke zu bezeichnen, die sich das Recht zur Kritik nehmen und damit ihren nonkonformistischen Mut beweisen. Sie erschreiben sich die Legitimität ihrer Kritik durch die Distanzierung von einem Kollektivsubjekt, zu dessen fiktionaler Existenz sie durch Distanzierung beitragen. Diese paradoxe Redeweise produziert ein kulturelles Surplus. Denn gelten sie erst einmal als kritische Meinungsmacher, werden ihre Meinungen allein deswegen von allen Seiten zur Kenntnis genommen, weil sie als solche gelten.

Ideologiekritik der von Pohrt repräsentierten Art ist allerdings hilflos, wenn sie dem denunzierten Verrat gegenüber allein auf den Begriffen von Ausbeutung, Klassenkampf, Revolution und Imperialismus zu bestehen weiß. Sie verkennt, daß gerade das Spiel der feuilletonistischen Rede - genau wie dies Greiner in der zitierten Bemerkung befürchtet - die Linke neu erzeugt. Denn der Surplus der Kritik an der Linken läßt sich nur weiter gewinnen, wenn »die Linke« im Spiel selbstreferentieller Kritik und Gegenkritik weiter erzeugt wird. Und so plädiert die für ihre »selling-out-Prakti- 
ken« vielgescholtene Cora Stephan für die Erhaltung linker Gesellschaftskritik und dafür, daß die Demokratie die Linke als ihren Feind brauche (Stephan 1993, 36).

Darüberhinaus nimmt Ideologiekritik den rationellen Kern der kulturellen und politischen Vorgänge nicht ernst, die zu einer Verschiebung des intellektuellen Sinnhorizonts geführt und aus vormaligen Linksradikalen iiberzeugte Anhänger der Marktwirtschaft, aus Avantgardisten des revolutionären Umsturzes loyale Verfassungspatrioten, aus Rohrstockpaukern der ML-Schulungen reformierte Oberlehrer einer kommunikativen Zivilreligion gemacht haben. Diese Verschiebungen sind Ergebnis durchaus selbstkritischer Einsichten von Intellektuellen in ein fragwürdiges, weil unkritisches Verhältnis zur Kritik der Gesellschaft und zu einer intellektuellen Redeposition, die durchaus autoritäre Züge trug und trägt. Doch werden die richtigen Einsichten in die autoritäre Praxis von Intellektuellen ihrerseits wieder ihres gesellschaftskritischen Gehalts beraubt - wie im weiteren zu zeigen sein wird.

\section{II.}

Die selbstkritische Überprüfung der Redeposition von linken, gesellschaftskritischen Intellektuellen ist ein wichtiger Beitrag zur Emanzipation von selbstorganisierter Subalternität. Denn in diese Redepositionen schleichen sich unversehens usurpatorische Machtansprüche ein. »Die Verbre-chen der Linken im Lauf dieses Jahrhunderts haben viel mit intellektueller Arroganz zu tun.« (Walzer 1992, 296; vgl. Althusser 1978, 55) Im Namen ihres Wissens über die Vernunft, die gesellschaftliche Totalität und die wirklich allgemeinen Interessen der Menschheit und der Gesellschaft droht linken Intellektuellen die Gefahr, ihre partikulare Redeposition mit dem Ganzen zu verwechseln. Es sind ja sie, die für sich in Anspruch nehmen, der Gerichtshof der Vernunft zu sein, vor dem sich Interessen und Bedürfnisse als richtige und falsche, partikulare und allgemeine, Wissen als wissenschaftlich oder unwissenschaftlich, Verhaltensweisen als fortschrittlich oder rückschrittlich, Zielsetzungen als Befreiung der Menschheit oder Beitrag zur Unterdrückung ausweisen müssen. Der Anspruch, den Standpunkt einzunehmen, von dem aus sich die Totalität der Gesellschaft erkennen lasse, erweist sich als elitär und - der Tendenz nach - als totalitär, insofern behauptet wird, daß nur die Intellektuellen diesen Standpunkt der Totalität einnehmen können, die über eine privilegierte Theorie verfügen, welche gleichzeitig erklärt, daß alle anderen dazu eben nicht tähig sind. Auf diese Machtwirkungen linker Theoriebildung zielt Foucault, wenn er dem Marxismus und der Psychoanalyse ihre Wissenschaftlichkeit vorwarf. Welches 
Wissen von unten werde disqualiziert, so fragt er, welche Macht angestrebt, welche Redeposition usurpiert mit dem Anspruch, im Namen der Wissenschaft zu sprechen, gar einer Wissenschaft, die die Entwicklungsgesetze der Geschichte, die Ziele ihres Verlaufs und die eigentlichen Interessen der Akteure zu kennen beansprucht. Foucault zufolge handelt es sich um Machtwirkungen, die $\gg$ das Abendland seit dem Mittelalter der Wissenschaft zugewiesen und jenen vorbehalten hat, die einen wissenschaftlichen Diskurs halten« (Foucault 1978a, 64).

Foucault zufolge ergab sich der Status des universellen Intellektuellen historisch aus seinem besonderen Zugang zur schriftlichen Kultur. Da sich dieses materielle Verhältnis seiner Ansicht nach seit einigen Jahrzehnten schon veränderte, würde der universelle Intellektuelle allmählich von einem neuen Typ des Intellektuellen abgelöst, dem spezifischen Intellektuellen, dem Spezialisten, der allein im Sinne seiner Spezialkenntnisse in die politische Diskussion eingreife. Mit dem Hinweis auf den Protest von Atomwissenschaftlern schreibt Foucault: »Der Intellektuelle wurde damals (nach dem Zweiten Weltkrieg; AD) wohl zum ersten Mal von der politischen Macht nicht mehr wegen seines allgemeinen Diskurses verfolgt, sondern wegen seines Wissens: Das war die politische Gefahr.« (Foucault 1978b, 82) Mit Intellektuellen meint Foucault hier, wie vor ihm auch Sartre schon, Physiker, Biologen und Mediziner.

Foucaults Diagnose scheint sich zu bestätigen. Im Zusammenhang mit der Entstehung der neuen sozialen Bewegungen entwickelte sich ein weitläufiges Gegenexpertentum. Doch mehr noch geht mit der feuilletonistischen Verabschiedung von Utopie und Geschichtsphilosophie der Verzicht auf den Anspruch einher, im Namen einer zukünftigen Freiheit und Wahrheit eine elitäre Redeposition einzunehmen, von der aus das falsche Bewußtsein der anderen zu entlarven wäre. Als kritische Reaktion auf solche Machtprozesse gibt sich die postsozialistische, postmoderne Intelligenz pragmatisch. Sie optiert dafür, sich von Fall zu Fall zu orientieren und die Vorstellung eines Jenseits der gegenwärtigen historischen Epoche fallen zu lassen: »Keine linke Kritik und keine linke Utopie werden mehr das ganz Andere zu bringen vermögen, also weder den Sozialismus noch gar das Himmelreich auf Erden, sondern bestenfalls wieder »Verhältnisse schaffen...« (Joschka Fischer) Die Intellektuellen fordern von sich selbst, sich weltimmanent, diskursiv und pluralistisch an der »kalten Gegenwart« der Demokratie zu orientieren, ohne $\gg$ Vision, ohne Utopie, ohne Grandiosität, ohne Transzendenz « (Cora Stephan) - auch wenn sie selbst $\mathbb{Z}$ weifel haben, ob ohne utopische Transzendenz sich auch nur Realpolitik machen ließe. Konsequent überlassen sie sich libertär dem »antiautoritären Potential der Marktveranstaltung Kapitalismus« (Thomas Schmid). Dies alles wird als 
angemessene Ernüchterung von Intellektuellen gesehen, die selbstkritisch auf ihre früher erhobenen kulturellen Machtansprüche, ihre privilegierte Redeposition und ihre usurpatorische Moralität verzichten und nun in eigenem Namen als Teilnehmer in einer offenen Diskussion ohne Ende und ohne Anspruch auf einen »letzten Sieg« das Wort zu ergreifen vermeinen. Unverkennbar hat dieses neue Muster einen emanzipatorischen Aspekt. Allerdings verändert sich auch der Charakter der Kritik - sie wird unkritisch, ja teilweise sogar antiintellektualistisch, insofern die Redeposition der Intellektuellen innerhalb gesellschaftlicher Kontexte aufgrund einer gewissen Selbstgerechtigkeit erneut aus dem Blick gerät und mithin die Machtwirkungen dieser neuartigen Form von Intellektualität.

Auf die Risiken dieses neuen Typs des Intellektuellen wies bereits Foucault hin. Er könne als Experte von den Parteien und Verbänden für deren Zwekke manipuliert werden und mangels einer Globalstrategie und gesellschaftlicher Unterstützung seine Kämpfe nicht weiter fortführen (vgl. Foucault 1978b, ebd.). Im Fall des Atomphysikers liegt das Kriterium der Kritik nahe. Denn es ist - wie auch Foucault zugestehen muß - sein spezifischer Gegenstandsbereich und die damit zusammenhängende Gefährdung der gesamten Menschheit, die ihn aus der Enge des Fachgebietes hinausführt und wiederum zum universellen Intellektuellen macht. Darüberhinaus hat Foucault in seiner Kritik an den universellen Intellektuellen des traditionellen Typs nicht thematisiert, was mit ihnen zukünftig geschieht. Denn sie sind weiterhin durch ihr privilegiertes Verhältnis zur Schrift ausgezeichnet und lehren und lernen weiterhin an Schulen und Hochschulen.

Foucaults Diagnose scheint ein performativer Erfolg geworden zu sein, insofern diese traditionellen Intellektuellen nun gleich ihm als universelle Intellektuelle permanent ihr Ende beschwören, ohne doch jemals spezifische Intellektuelle werden zu können. Indem sie das Kriterium der Kritik an ihrer Universalität übernehmen, verschieben sie die Kritik. Sie kritisieren, und zwar durchaus in einer sehr traditionellen, ideologiekritischen Weise, daß alle anderen Intellektuellen noch immer in einem falschen Bewußtsein von ihrer professionellen Rolle befangen seien. Die Kritik an den Intellektuellen ist keine Kritik an den Intellektuellen als Wortführern und Parteigängern bestimmter sozialer Entwicklungstendenzen mehr. Indem sich die Kritik allein auf die autoritäre Redeposition der Intellektuellen selbst konzentriert, geht die Kritik ein affirmatives Verhältnis zur Gegenwart ein. Die selbstreferentielle Kritik erweckt den falschen Eindruck, daß gesellschaftliche Macht verschwinden würde, wenn Intellektuelle die von ihnen selbst ausgeiibte ablehnen. De facto können Intellektuelle aber gar nicht anders, als in ihrer Rede bestimmte soziale Parteien zu repräsentieren, soziale Entwicklungen zu begünstigen, zu korrigieren oder zu verwerfen. Es geht un- 
ter den Bedingungen einer kapitalistischen gesellschaftlichen Arbeitsteilung mit der Trennung von geistiger und körperlicher Arbeit nicht anders, als daß die Intellektuellen eine paradoxe Rede führen. Diese ist notwendig selbstreferentiell und kann sich ausschließlich nur auf das intellektuelle Feld und die Äußerungen anderer Intellektueller beziehen: es wird gelesen, zitiert, interpretiert, kommentiert, beobachtet, verallgemeinert, kritisiert aber immer nach den Standards, den Regeln und Kriterien des sozialen Feldes, in dem die Intellektuellen als Wissenschaftler, Autoren, Journalisten etc. aktiv sind. Sie können aus diesem Feld nicht individuell heraustreten, weil es die soziale Bedingung ihrer Existenz ist und sie in spezifischer Weise in ein Verhältnis zur Gesellschaft setzt. In diesem Feld produzieren sie durch ihre Rede als sozialer Praxis symbolische Macht und kulturelle Herrschaft in der Form bestimmter allgemeingültiger Begriffe, relevanten Wissens, legitimer Kultur. Die Selbstreferenz läßt sich als solche durch Kritik im intellektuellen Feld nicht abschütteln. Die Kritik der von Intellektuellen ausgeübten Autorität ist daher häufig nichts anderes als ein Element der Machtverschiebung von einer Intellektuellengruppe auf eine andere oder ein Merkmal der Transformation von Intellektuellengruppen, die auf andere und neue Weise kulturelle Hegemonie auszuüben versuchen.

Die Schwäche des Kritikparadigmas von Foucault ist demnach, daß es einem normativen Begriff des Intellektuellen einen anderen normativen Begriff entgegensetzt und damit die Dynamik des kulturellen Feldes als ein Kräftefeld intellektueller Tätigkeiten unterschätzt. Doch in dem weitverzweigten Netz von konflikthaften öffentlichen Diskussionen und kulturellen Auseinandersetzungen in der Zivilgesellschaft gibt es kein Machtvakuum. Es entstehen immer wieder neue Formen von Intellektualität und Redepositionen, die im Namen einer Allgemeinheit das Wort führen und damit den Anspruch auf selbstkritische Selbstbeschränkung überholen, relativieren und - durch ihren kritischen Anspruch hindurch - einen neuen affirmativen Charakter annehmen können.

In der Zivilgesellschaft entstehen ständig neue Gruppen von Intellektuellen mit jeweils neuen Institutionen und neuen Netzwerken. Sie bilden eigene Verständigungsformen und Kritikstrategien heraus. Es kann keine Rede davon sein, daß die Zeit des universellen, traditionellen Intellektuellen vorbei ist. Vielmehr ändern sich zumeist krisenhaft die Konfigurationen intellektueller Netzwerke, indem sich die Intellektuellen molekular aus einer intellektuell verbindlichen Gruppe mit ihren Kulturtechniken, Weltsichten und Lebensformen lösen, neue Verbindungen knüpfen und neue Verhälínisse zu ihrer Tätigkeit eingehen. Die Wirkungen - auch wenn dies in winzigen, feuilletonistischen Schritten erfolgt und auf den ersten Blick anscheinend nicht ernst zu nehmen ist - sind historisch nachhaltig, denn das gesamte 
kulturelle Spektrum ändert sich. Es entstehen neue, kulturelle Machtverhältnisse zwischen intellektuellen Gruppen. Eine der wichtigen Einsichten, die Gramscis Analysen der Zivilgesellschaft erbracht haben, ist, daß es theoretisch unergiebig ist, Intellektuellen Abweichungen, Verrai oder Selbstbezüglichkeit vorzuwerfen. Der Blick wäre vielmehr auf die Gesamtheit des intellektuellen Feldes zu richten, auf dem die verschiedenen intellektuellen Gruppen mit ihrer Existenz und ihren Transformationen einen historisch spezifischen Ereignisraum des konflikthaften Handelns konstituieren. Alle diese Intellektuellenparteien beobachten sich wechselseirig und die gesellschaftlichen Prozesse, die sie kommentieren, interpretieren und analysieren. Sie kritisieren die jeweils anderen Parteien und sich selbst, um Fehler festzustellen, Perspektiven auszumachen und die anderen in ihren Handlungsmotivationen zu demoralisieren. Kritik als solche und der Mut zur Kritik ist also kein Privileg einer besonderen Intellektuellenpartei. In der Polarisierung und Parteibildung bilden sich unterschiedliche Formen von Kritik aus. Die Frage ist nun, ob es Wege gibt, das sich in der intellektuellen Rede linker Gesellschaftskritik entfaltende autoritäre Potential durchaus im Sinne Foucaults - konsequenter zu kritisieren, ohne damit den Raum für eine affirmative Form von Allgemeinheit zu öffnen.

III.

Wenn die Glaubwürdigkeit gesellschaftskritischer Begriffe und Theorien verloren ging, stellen sich erneut die bereits von Horkheimer aufgewortenen Fragen: woraus sich der verpflichtende Charakter einer Gesellschaftskritik ergibt und woraus sie ihre Kraft bezieht. Allerdings kann nicht mehr. wie im Fall der Kritischen Theorie, davon ausgegangen werden, daß es keine Kritik mehr gebe und die Intellektuellen alle nichts anderes tun. als den Status quo durch Affirmation zu befestigen. Es gibt Kritik als kritische Haltung der Entunterwerfung, als Haltung, sich nicht so und nicht dermaBen regieren zu lassen (vgl. Foucault 1992, 11). Kritik ist konstitutiv für die Zivilgesellschaft, die sich in den von Intellektuellen organisierten Prozessen der Kritzk und Selbstkritik permanent transformiert. Doch auch die Kritik ändert ihre Formen, ihre Inhalte und ihre Ziele. In einigen seiner Veröffentlichungen hat Michael Walzer den Versuch unternommen, Idealtypen der Gesellschaftskritik herauszuarbeiten, indem er die Performanz. von Intellektuellen zum Gegenstand einer Analyse macht. Er unterscheidet drei Typen und Praktiken der Gesellschaftskritik: die Entdeckung, die Erfindung und die Interpretation. Die Entdeckung und die Exfindurg sind zwei Formen der Gesellschaftskritik, die Walzer ablehnt, weil sie von der Vorstellung geleitet sind, die Kritik einer Geselischaft müsse entweder von 
einem distanzierten Ort irgendwo dort draußen kommen oder sie bestärke den Status quo. In beiden Fällen wird erwartet, daß die Kraft der Kritik sich aus höheren Prinzipien ableite. Im Fall der zehn Gebote tut er dies im Namen Gottes. Die moralischen Prinzipien stehen in scharfem Gegensatz zu den überlieferten Vorstellungen und Praktien. Sie stellen einen bis dahin unbekannten Kontinent dar, dessen Landkarte Gottes Diener kennt. Das Modell der Entdeckung hat autoritäre Konsequenzen, denn es wird kaum jemand behaupten, die richtigen Moralprinzipien gefunden zu haben, ohne den Versuch zu unternehmen, sie auch gegen alle durchzusetzen. In säkularisierter, philosophischer Form wird dieses Modell im Fall einer auf Naturrechte gestützten Kritik praktiziert. Auch der Marxismus wird von Walzer diesem Modell zugerechnet.

Der zweite Typ der Gesellschaftskritik, zu dem Walzer Philosophen wie Rawls und Habermas rechnet, ist der der Erfindung. Der Erfinder trete an die Stelle Gottes und schaffe eine neue moralische Welt. Ein Motiv für diese Tätigkeit sei, daß die Kritiker die moralische Welt für unzulänglich halten. Die Autorität dieser Kritikstrategie solle sich aus dem Ziel ergeben, nämlich einem »gemeinsamen Leben, in dem Gerechtigkeit, politische Tugend, gutes Leben oder irgendein anderer solcher Grundwerte verwirlicht wäre « (Walzer 1990, 18). Daß jeder der objektiven Wahrheit und dem Verfahren, an dem er virtuell beteiligt sein soll, auch zustimmen kann, soll ihn gleichzeitig verpflichten. Gegen beide Formen der Gesellschaftskritik hat Walzer einen zentralen Einwand. Seiner Ansicht nach scheitern diese Kritikstrategien daran, daß sie suggerieren, sie seien die alleinige und entscheidende Form der Kritik. Sie müssen den pluralen Charakter von Moralprinzipien und Kritikstrategien ebenso ignorieren wie die Tatsache, daß es eine endlose Zahl von Entdeckern und Erfindern gibt. Walzer gibt damit einen Hinweis auf den interessanten Vorgang in der Moralphilosophie, daß nämlich Versuche, Gesellschaftskritik auf dem Weg moralischer Normbegründung eine Grundlage zu geben, sich selbst ad absurdum führen, weil es zu einem Überbietungswettbewerb kommt, jeweils noch bessere und noch tiefer liegende Begründungen allgemein verpflichtender Prinzipien zu finden. Des weiteren scheitern Walzer zufolge Moralerfindungen daran, daß sie ihre Moralprinzipien nicht gegen die Deutungen und Interpretationen durch die gesellschaftlichen Akteure immunisieren können; mit anderen Worten, sie sind letzllich immer schon Interpretationen.

Dieser Befund ist für Walzer Anlaß zu gesellschaftskritischer Nüchernheit. Es braucht keine Gesellschaftstheorie, keine entdeckten und erfundenen, keine begründeten Moralprinzipien für die Kritik. Gesellschaftskritik ist immer schon da. Sie muß nicht warten, bis Philosophen die tieferen Gründe gefunden haben, auf denen die Kritik sicher aufruht. Ihre Prinzipien fin- 
den sich in der moralischen Welt, in der wir leben, und die uns mit allem »versorgt, was wir benötigen, um ein moralisches Leben zu führen - die Fähigkeit zur Reflexion und Kritik eingeschlossen« (ebd., 31; vgl. 29). Die vorhandene und uns deswegen auch bereits verpflichtende Moral wird von uns im Lichte neuer Konflikte und Interessen interpretiert. Fortschritte der Moral ergeben sich nicht durch Entdeckung oder Erfindung, sondern im Streit kontroverser Interpretationen, durch Gesellschaftskritik, durch die »zuvor aus den alten Prinzipien ausgeschlossene Männer und Frauen « in den Geltungsbereich der tradierten Moralprinzipien integriert werden (ebd., 37). Die von uns geteilte Moral beantworte niemals alle moralischen Fragen und sei immer inkonsistent. Deswegen habe sie auch immer einen für Macht und Herrschaft potentiell subversiven Charakter. Sie gibt nämlich den Gesellschaftskritikern die Prinzipien an die Hand, mit deren Hilfe sie daran erinnern können, daß es zwischen ihnen und unserem alltäglichen Leben Brüche gibt. Kritik ist also immer möglich, weil nicht nur die Herrschenden, sondern alle sich rechtfertigen wollen. Nicht auf die Prinzipien komme es an, sondern auf den Prozeß der Interpretationen, der gleichzeitig wiederum alle verpflichte sich weiterzustreiten. »Es gibt eine Überlieferung, einen Korpus moralischen Wissens; und es gibt eine Gruppe von Weisen, die sich streiten. Etwas anderes gibt es nicht. Keine Entdeckung oder Erfindung kann den Streit beenden « (ebd., 42).

Damit bestreitet Walzer die Notwendigkeit, daß ein Kritiker zu den gesellschaftlichen Verhältnissen auf Distanz gehen sollte. Er ist einfach ein Interpret unter vielen anderen. Der Kritiker, der Abstand gewinnen und sich selbst und anderen den Eindruck vermitteln möchte, er sei der große Einsame, der moralisch Integre, ästhetisch besonders Sensible, für jedes Argument Offene, der Wahrheit zugetan und bereit für sie zu sterben, kurz der Intellektuelle als Märtyrer und Held - diese Figur ist für Walzer der Entdecker und Erfinder. Ähnelt in Walzers Modell die Tätigkeit des Entdeckers der der Exekutive, die Tätigkeit des Erfinders der der Legislative, so nimmt der von ihm favorisierte Gesellschaftskritiker die Rolle eines lokalen Richters wahr, des mit

»seiner Gesellschaft verbundenen Kritikers, der seine Autorität aus der Auseinandersetzung mit seinen Gesellschaftsgenossen gewinnt (oder auch nicht gewinnt) - der mit Leidenschaft und ohne Unterlaß, manchmal mit hohem persönlichen Risiko (auch er kann ein Held sein) Einspruch erhebt, protestiert und Einwendungen macht. Dieser Kritiker ist einer von uns«(49).

Die Haltung des nüchternen Pathos, die Michael Walzer für sein Modell des subversiven Gesellschaftskritikers vorsieht, ist sympathisch. Sie nimmt die selbstkritischen Erfahrungen vieler linken Intellektuellen auf, die zu Recht versuchen, sich selbstkritisch gegen den Objektivismus ihrer kritischen Haltung und die Machtwirkungen ihrer eigenen Rede zu wenden. 
Gegen geschichtsphilosophisch begründete pessimistische Erwartungen geben die Hinweise auf jahrtausendealte Praktiken der Gesellschaftskritik, deren archetypische Modelle in der Bibel ausgearbeitet vorliegen, das beruhigende Gefühl, daß Kritik auch weiterhin möglich sein wird. Doch sehe ich eine Reihe von Problemen.

Wenn Walzer davon spricht, daß die Ressourcen für die Kritik da sind, erwartet er implizit, daß Kritik einer inneren Optimierungslogik folgt. Kritik steht für den Anspruch, Ausgeschlossene zu inkludieren. Doch handelt es sich um eine einseitige Festlegung von Kritik. Es gibt kritische Praktiken, die auf den Ausschluß zielt. Dies gilt für den Liberalismus, der Freiheit und Gleichheit für widersprüchlich und die Durchsetzung der Gleichheit für totalitär hält. Dies gilt für die Neue Rechte, die für sich selbst beansprucht, den Reedukationskonformismus der westlich orientierten politischen Klasse zu kritisieren. Aus dieser Praxis von Kritik und Gegenkritik resultiert, daß Ergebnisse einer Kritik auch wieder rückgängig gemacht werden. Soll der Prozeß der Kritik nicht autoritär stillgestellt werden, dann ist es richtig, davon zu sprechen, daß Auseinandersetzungen um die Entwicklung von Gemeinwesen nicht zu beenden sein werden, daß es keinen »endgültigen Sieg geben wird (Walzer 1992a, 297). Dies meint nicht nur, daß neben den Diskussionen auch die Kämpfe in alle Ewigkeil weitergehen (vgl. Walzer 1992b, 62). Auch die je historische Form von Kritik wird außer Acht gelassen: der Gesichtspunkt also, welche Verhältnisse die Kritik eingehen muß. Walzer geht von der Ewigkeit bestimmter Konstellationen aus: immer wieder kritisiert der Kommunitarismus den Liberalismus (Walzer 1993, 170); immer wieder von neuem nuß um die Grenzlinie zwischen Markt und Staat »gekämpft« werden; seit biblischen Zeiten gibt es die Entdecker, Erfinder und Interpreten. Die Rolle des Sisyphos mag zur Weltgeschichte gehören - was nur ein weiteres Merkmal für die Zwanghaftigkeit von Klassengesellschaften und den geringen Grad ihrer Freiheit wäre -, doch ist damit noch nicht befriedigend beantwortet, warum immer bestimmten sozialen Kategorien von Individuen diese Rolle ausschließlich zufältt. Walzers Hinweis auf die Pluralität unserer Gesellschaften als Grundlage für die Vielfalt von Kritik und Gegenkritik ist kein überzeugendes Argument. Moderne Gesellschaften schließen sich - zumindest ihren normativen Ansprüchen und ihren Selbstbeschreibungen nach - nicht (oder nur selten) $a b$, sind also offen, kritikfähig und plural. Doch auch diese Pluralität und Offenheit kann verschiedene Formen ausbilden. Kritisch läß sich darauf hinweisen, daß es sich eben um eine bestimmte gesellschaftiche Selbstbeschreibung handelt, dab moderne, komplexe Gesellschafter eben nicht plural genug sind und nur existieren können, wenn sie die Pluralität und Offenheit vielmehr durch Klassenmerkmale, staatliche 
Zugehörigkeit, durch ethnische und geschlechtliche Identitäten, durch die Mauern der Ausdifferenzierung - die Walzer mit staatlicher Hilfe gezogen und geschützt wissen möchte (vgl. Walzer 1992b, 60) - immer wieder von neuem einschränken. Es ist uns heute nicht möglich, über tiefgreifende, die kollektiven Lebensweisen bestimmende gesellschaftliche Verhältnisse entsprechend unseren vielfältigen und unterschiedlichen Interessen und $\mathrm{Be}$ dürfnissen zu entscheiden. Eine richtige Gesellschaft, so die Überlegung Horkheimers und Adornos, zeichne sich dadurch aus, daß sie wirklich pluralistisch sei. Allein der Hinweis auf Pluralität und Kritik reicht also nicht hin, vielmehr müßte Walzer Kriterien für die Güte der Form der Kritik und des Pluralismus ausweisen.

Zweitens bezweifle ich den demokratischen Charakter des Interpretationsbegriffs. Wir alle - so Walzer - interpretieren und setzen uns über gemeinsam geteilte Werte und Normen auseinander, in denen wir uns gleichzeitig unserer kollektiven Identität versichern. Doch der Gesellschaftskritiker wird von Walzer als der Weise verstanden, als der professionelle Kritiker, der qua amtlicher Autorität über die Gesellschaft richtet, er wird als Richter oder Advokat bestimmt. Kritik soll es deswegen geben, weil es Poeten, Geschichtenerzähler, Geschichtsschreiber und überhaupt Schriftsteller gibt und immer schon gegeben hat. Hier nimmt die Rückführung auf archaische Paradigmen der Gesellschaftskritik selbst einen konservativen Charakter an. Gegen Walzers Argument spricht, daß es Kritiker gibt, deren Kritik nicht durch den privilegierten Zugang zum Wort und zur Öffentlichkeit bestimmt ist oder sich sogar gegen diese richtet. Mit seinem Professionalismus aber bestärkt Walzer eine herrschaftlich organisierte Form gesellschaftlicher Arbeitsteilung, die zwangsläufig einen undemokratischen Charakter hat (vgl. auch Buchstein/Schmalz-Bruns 1992, 395). Gleichfalls ist die historische Konstruktion fragwürdig, ob es tatsächlich in biblischen Zeiten schon Kritik gegeben hat. So vertritt Foucault die historisch sehr viel konkretere Ansicht, daß sich Kritik als Haltung erst mit den religiösen und staatlichen Regierungspraktiken zu Beginn des bürgerlichen Zeitalters entwickelte (vgl. auch Koselleck 1979). Walzer folgt dem unter Intellektuellen verbreiteten humanistisch-idealistischen Mißverständnis, sich durch ihre selbstbezüglich Beschäftigung mit theoretischen Traditionen selbst den Status einer ewigen Kaste zuzubilligen. Entsprechend zweifelt Walzer auch nicht am Interpretationsbegriff, der von der Universalität und Kontinuität des Sinns ausgeht, der - entsprechend einem theologisch-juridischen Modell - immer nur neu interpretiert wird. Letztlich gilt ihm die Bibel als das Buch der Bücher, in dem sich jener Sinn ebenso finden läßt wie die Techniken für die Textexegese, den Umgang mit der Tradition und die kritische Haltung der Intellektuellen. Nichts Neues gibt es mehr, sondern jede 
Interpretation reichert nur die Ewigkeit dieses Sinnkosmos an. Dieser und die ihn konstituierenden Kulturtechniken der professionellen Intellektualität und der Interpretation werden von Walzer aber selbst nicht angezweifelt. Kritiklos wird die »Ewigkeit« dieser historischen Tradition hingenommen und durch Interpretation selbst immer von neuem in ihrer Klassizität bestätigt.

Drittens weist Walzer mit seiner These, daß Gesellschaftskritik nicht auf Distanz und von draußen kommen sollte, auf ein Problem hin, doch bestimmt er die gesamte kritische Praxis und die Wahrheitspolitik von Intellektuellen nicht angemessen. Damit geht auch eine falsche Stilisierung intellektueller Strömungen einher. Als Paradigma einer Gesellschaftskritik auf Distanz gilt Walzer die marxistische Tradition. Kritik von draußen sei undemokratisch. Doch empirisch ist dies - wie im Fall Marcuses (vgl. Walzer 1991, 251) - unzulänglich beschrieben, denn die Kritik von draußen ist immer eine Kritik von innen; sie kann bei vielen Gesellschaftsmitgliedern auf hohe Resonanz stoßen, die sich gegenüber dem Normalitätsdispositiv der herrschenden Gesellschaft ablehnend verhalten. Daß sie diesen Bruch in der Normalität ausarbeitet, kann demokratisierende Wirkung haben. Walzer kann gesellschaftstheoretisch nicht erklären, warum sich historisch die Kritikpositionen von außen immer wieder ergibt und erneuert, sondern möchte sie nur normativ vom intellektuellen Feld verdrängen und ausschließen - er möchte doch einen letzten, normativ verbürgten Sieg über die Konkurrenten im intellektuellen Feld. Daß seine eigene Rede gerade mit ihrem Anspruch auf Pluralität die Ausgrenzungslogik reproduzieren muß, entgeht Walzer, weil er sich einem gesellschaftstheoretischen Begriff der Zivilgesellschaft und des intellektuellen Feldes verschließt.

Doch auch in einer anderen Hinsicht hat Walzer nicht recht. Gegen den Voluntarismus und Autoritarismus von Utopien gerichtet, wurde von Marx mit dem Anspruch auf Wissenschaftlichkeit die Vorstellung einer nachvollziehbaren, einsichtigen und argumentativen Kritik verbunden. Der Kritik-Begriff, so wie er von Marx und vielen seiner Nachfolger entwickelt wurde, ist der einer immanenten Kritik - immanent allerdings nicht im Sinne einer einheitlichen, gemeinsam geteilten, »unserer«, sondern einer gespaltenen und unversöhnten Welt. Marx' Kritik läuft darauf hinaus, daß die klassengespaltene moderne Gesellschaft nicht wirklich immanent werden kann, sondern sich immer im "ewigen " Sinnhorizont der Theologie bewegt und an Transzendenz orientiert. Utopie ist die einzige und theologische Form, in der die bürgerliche Gesellschaft ihre Zukunft entwirft: als Versöhnung ihrer Normen mit ihrer Wirklichkeit. Wollte sie ihre Immanenz bewerkstelligen und ihre Zukunft abschließen, würde sie zwangsläufig repressiv. Sie ginge in Totalitarismus über. So ist es aus der Sicht von 
Marx nur eine Bestätigung der Dilemmata des Liberalismus, wenn seine VertreterInnen zugestehen, daß der Totalitarismus sich nur um den Preis verhindern läßt, daß auch Demokratie nicht verwirklicht werde. Selten pointiert formuliert dies Chantal Mouffe, wenn sie, ganz unkritisch, sagt: "Ist es nicht paradox, daß die pluralistische Demokratie nur wegen der Unmöglichkeit ihrer Vollendung existieren kann?« und Michael Walzer zustimmend bemerkt: »Ja, Politik ist langwierig.«(Walzer 1992a, 296) Ziel der Kritik von Marx war es, auf die Bedingungen hinzuweisen, unter denen das Zusammenleben der Individuen sich endlich aus dem Horizont der Theologie in allen ihren Formen (Gottes, des Staates, der Ware, des Geldes) herausbewegen und immanent werden konnte - und dies, ohne daß es zur Herausbildung einer identitären Gesellschaft käme. Der geschichtliche Prozeß wird gerade deswegen nicht als Heilsgeschehen hin zur Erlösung von den Übeln und Einlösung schon bekannter Normen betrachtet, sondern als ein prozessierender Widerspruch, der sich durch Aneignung fremder Arbeit und Beherrschung, durch Kritik, Widerstand und Emanzipation fortbewegt. Das Ziel ist nicht die Transzendenz, das Jenseits, die Abschaffung von »Verhältnissen«, sondern die Beendigung dieser bestimmten Form von Verhältnissen und Widersprüchen und die Beseitung aller Formen von quasi transzendenten Mächten, die die Entscheidungsfreiheit der Menschen verhindern.

\section{$\mathbb{I V}$.}

Die feuilletonistischen Beobachtungen thematisieren ebenso wie Foucault und Walzer eine Veränderung der Intellektuellen im Verhältnis zu sich selbst. Sie überprüfen die Machtwirkungen ihres Wissens und ihrer Redeposition. Ihre kritische Haltung zielt darauf, sich nicht mehr führen zu lassen und nicht selbst aktiv zu führen, sondern RednerInnen unter den vielen anderen zu sein, antiautoritär gegen jede Machtanmaßung der Wahrheit, emanzipiert davon, sich den Sinn der Gegenwart von einer mächtigen Zukunft oder von der Wucht der sozialen Gesetze der Vergangenheit vorschreiben zu lassen. Diese Kriterien der Kritik und Selbstkritik sind Maßstäbe des intellektuellen Selbstverhältnisses, die in ihren Konsequenzen für viele der Begriffe in der linken Theorietradition ernst genommen werden müssen, gerade weil sie sich in der Kritik daraus ergeben haben und in vielen Hinsichten autoritäre Momente treffen. Doch auf paradoxe Weise ignorieren diese Kritiken die Machteffekte ihrer eigenen neuen Redeposition und der Form von Selbstkritik. Damit meine ich nicht allein ihr Vertrauen in den Markt, ihre Besinnung auf die Würde des Staates, ihre Achtung vor den Werten, ihre Beschwörung des Pluralismus der massenme- 
dialen Öffentlichkeit - vielfach überzogene Reaktionen auf die Aporien der traditionellen Ideologiekritik, die aufs Neue die gesellschaftlichen Widersprüche ausblenden. Die Selbstkritiken der Intellektuellen unterstellen und sind damit einer gewissen konservativen Tradition verpflichtet -, daß es allein ihre Rede und ihre Vernunft- und Wahrheitsansprüche gewesen seien, die als indirekte politische Gewalt letztlich das staatliche Monopol auf Allgemeinheit usurpieren würden. Die Kritik ist zu Recht selbstbezüglich gegen diese Funktionen gerichtet. Doch ist sie immanent und deswegen begrenzt. Von Foucault war diese Begrenztheit der Kritik gewollt. Er zielt auf das Ethos einer Kritik der Gegenwart und der »uns gegebenen Grenzen«, verbunden mit dem Experiment der Möglichkeit ihrer Überschreitung (Foucault 1990, 53). Um die Fehler der Usurpation zu vermeiden, soll sich die Kritik auf eine situative, eine partielle Kritik beschränken, durch die Intellektuelle ein neues Verhältnis zu sich selbst eingehen mit dem Ziel, sich nicht so, sich nicht dermaßen regieren und führen zu lassen (vgl. Foucault, 1992, 51ff.) - womit unterstellt wird, daß Regierung und Führung als soiche keinesfalls zweifelhaft sei. Doch Foucaults eigenes Freiheitsstreben, sein Anarchismus läßt ihn sich »versprechen«: es geht ihm auch um den Willen und die Haltung, sich überhaupt nicht regieren und führen zu lassen (ebd., 41). Die Kritik kann zunächst nicht anders als selbstbezüglich an den Grenzen des intellektuellen Feldes, also alles dessen, was Intellektuelle denken, wissen und tun, zu operieren. Thre antitheologische und antimetaphysische Bedeutung hat sie aber darin, daß sie auch die Grenzen als solche erkennt und den Versuch unternimmt, von innen heraus über die Grenzen dieser »metaphysischen« Gegenwart hinauszutreten und ihre eigenen Existenzgrundlagen zu thematisieren. Denn die Redeposition der Intellektuellen kann die Macht nur erzeugen aufgrund ihres Status als professionelle Intellektualität in der gesellschaftlichen Arbeitsteilung. Diesen Schritt geht Foucault nicht, obwohl er die brïderliche Annäherung an die Frankfurter Schule sucht (vgl. ebd., 25).

Die Kritische Theorie bestand auf immanenter Kritik in einem sehr radikalen Sinn. Es sollte nicht der Hinweis auf die Zukunft und die sozialen Gesetzmäßigkeiten in Anspruch genommen werden, weil dies letztlich nur an die konformistische Zustimmung zu einer tieferen und zukünftigen Macht appelliert. Gleichfalls lehnte sie es ab, ihre Kritik auf die Normen von Freiheit und Gleichheit zu stützen, denn schon längst hatten diese ihre Verpflichtungskraft und Bindungsfähigkeit für das Bürgertum verloren. Unter dem von der Arbeiterbewegung ausgeübten Druck, die Glücksversprechen der bürgerlichen Gesellschaft endlich wahr zu machen, so die nachhaltige Erfahrung der Vertreter der Kritischen Theorie, verzichtete das Bürgertum darauf, an diesen Normen überhaupt noch festzuhalten. Wie schon Marx in 
seiner Kritik an der Französischen Revolution und den Frühsozialisten, deutet auch Adorno Vorbehalte gegen Gleichheit an.

»Eine emanzipierte Gesellschaft jedoch wäre kein Einheitsstaat, sondern die Verwirklichung des Allgemeinen in der Versöhnung der Differenzen. Politik, der es darum im Ernst noch ginge, sollte deswegen die abstrakte Gleichheit der Menschen nicht einmal als Idee propagieren. Sie sollte statt dessen auf die schlechte Gleichheit heute ... deuten, den besseren Zustand aber denken als den, in dem man ohne Angst verschieden sein kann« (Adorno 1980, 114).

Der Intellektuelle als Kritiker soll immanent vorgehen, weil allein dies verhindert, usurpatorische Ansprüche zu erheben.

»Die objektive Verstelltheit des Besseren betrifft nicht abstrakt das große Ganze. In jedem Einzelphänomen, das man kritisiert, stößt man rasch auf jene Grenze« (Adorno 1977b, 793).

Eine von außen geführte Kritik hat ihrerseits keine Verbindlichkeit, wenn sie nicht in der Lage ist, die zu kritisierenden Interessen, Bilder, Begriffe und Denkweisen aufzunehmen und zu bearbeiten. Doch woran könnte eine solche Kritik ihren Maßstab haben, wenn alle immanenten Kriterien ihrerseits schon als Maßstab verworfen wurden, weil sie historisch entweder obsolet oder aber metaphysisch-autoritär sind? ${ }^{1}$

Adomos Kritikstrategie besteht in der Empfehlung, der Dialektik der Immanenz zu folgen. Nachdem die Intellektuellen sich als solche anerkannt haben und erkennen, daß ihr Ausgangspunkt die Immanenz ist, müssen sie, wenn sie die Kritik nicht voreilig und aus partikularistischen Motiven sistieren, weiterhin erkennen, daß die Immanenz ihres Denkens ein Zwang ist und die intellektuelle Betätigung auf das arbeitsteilige Feld intellektueller Objektivationen festschreibt und ihre Erkenntnisfähigkeit beschränkt. »Gerade der zur Sonderfunktion beschränkte Geist verkennt sich, dem eigenen Privileg zuliebe, als Absolutes.« (Adorno 1971, 20) Immanenz impliziert den Wahn von der Absolutheit des Geistes und der Reinheit des Intellekts. Intellektuelle nehmen für sich Intellektualität, das Wort, die Kultur, die Vergangenheit, den Geist, die Freiheit in Anspruch - als sei ihre Existenz und ihre Praxis nicht immer schon durch das Unrecht an denen gekennzeichnet, deren Arbeit sie sich nicht allein nur verdanken, sondern die sie mit ihrem Denken regieren und führen (vgl. Demirovic 1993). Kon-

1 In keinem Fall ist es der unter Linken beliebte direkte Schritt zum Wesentlichen - dem Weltmarkt, den kapitalistischen Produktionsformen, den liberaldemokratischen Institutionen etc. -, so als sei Kritik nur möglich, wenn sie sich mit vorweg definierten großen Dingen abgibt. Die bindende Wirkung der Analyse als Kritik springt nicht aus den analysierten Verhältnissen, und seien diese noch zu schreckerregend. Eine solche Annahme tendiert dazu, autoritär und antiintellektuell zu werden, da sie den Prozeß der intellektuellen Aneignung und die Auseinandersetzungen ums Relevante durchstreicht und das für wesentlich Gehaltene schlicht setzt. Die bindende Wirkung einer kritischen Analyse kann sich nur in der konkreten Auseinandersetzung mit dem intellektuellen Feld und der Dialektik der Begriffe einstellen (vgl. Adorno 1968, 33ff.). 
stitutiv ist selbst noch der demokratischen Rede der Intellektuellen die Teilhabe an sozialer Herrschaft und der Nutzen, den sie daraus ziehen, eingeschrieben. Und dies gilt gerade für die demokratisch-nüchterne Rede des Intellektuellen über den Intellektuellen, die auf alles Pathos und intellektuelles Geziere verzichtet und die geistige Arbeit mit dem Hinweis auf die in modernen, komplexen Gesellschaften liegenden Notwendigkeiten begründet. Die von Intellektuellen ausgearbeitete Theorie der Moderne wird so zur selbstreferentiellen Usurpation der gesellschaftlichen Rede - sie folgt der »ewigen « Tradition der Intellektuellen, ihre Rede durch ihre Rede zu privilegieren, indem sie in Anspruch nehmen, advokatorisch für andere zu sprechen, die im gleichen Atemzug unmündig gemacht werden.

Demgegenüber wird das Plädoyer für eine Selbstkritik der Vernunft bis auf weiteres der zeitgemäße Impuls der Kritischen Theorie bleiben. Dies meint, daß die Intellektuellen - wollen sie sich und ihre Kritik nicht für unmündig erklären - erkennen und analysieren sollen, worin das Wahnhafte und Autistische, die theologische und metaphysische Befangenheit ihres Denkens, ihres Leidens, ihrer Sensibilität, ihrer Erfahrungen, worin die Grenzen ihrer Erkenntnis, die Wiederkehr der immer gleichen Probleme und Überlegungen begründet ist. Der Weg aus der Immanenz der professionellen Intellektualität ist die Erkenntnis der metaphysischen Transzendenz der Intellektualität selbst als historisch spezifischem Ort der Rede professioneller Intellektueller. Sie ist Ergebnis der gesellschaftlichen Trennung von Regierten und Regierenden, derjenigen, die konzipieren, von denen, die ausführen. Diese Form der Selbsterkenntnis ihrer Tätigkeit, die das Denken, die Kultur, die Diskussionen als Öffentlichkeit und Zivilgesellschaft verselbständigt, folgt der Dynamik der Erkenntnis selbst und führt zu dem Selbstmißtrauen der Intellektuellen gegenüber ihrer Existenz, ihrer Vernunft und zu dem »subjektiven Wissen ums schlechte Ganze«. Immanentes Verfahren der Kritik mündet schließlich ein in ein Wissen von außen, das »jeden Augenblick in die Bestimmung des Gegenstandes mit eingeht« (Adorno 1977a, 28). Diese Dialektik von Innen und Außen darf nicht zugunsten einer Seite stillgestellt werden, bis schließlich diese Bewegung selbst sich als historisch beschränkte Bewegung herausstellt: »Am Ende wird der dialektischen Methode der Gegensatz der von außen und von innen eindringenden Erkenntnis selber als Symptom jener Verdinglichung suspekt, die anzuklagen ihr obliegt« (ebd.). Das Außen, auf das Adorno mit seiner Konzeption einer dialektischen Intellektualität zielt, ist die Bruchstelle einer Gesellschaft, die aufgrund ihrer Widersprüche nicht wirklich immanent zu werden vermag. In jener Bruchstelle, jener Nichtidentität deutet sich das Neue an. Es ist die Kritik der spezifischen Form der gesellschaftlichen Arbeitsteilung, die die Trennung von Führern und Ge- 
führten, von Sprechern und Repräsentierten, von Planenden und Ausführenden, von Immanenz und Transzendenz überholt und die gesellschaftlich selbstbestimmte Arbeitsteilung und Kooperation ermöglicht.

\section{Literatur}

Adorno, Th.W. (1971): Zur Metakritik der Erkenntnistheorie, Gesammelte Schriften 5, Ffm Adorno, Th.W. (1977a): Kulturkritik und Gesellschaft, Gesammelte Schriften, 10.1, Ffm

Adorno, Th.W. (1977b): Kritik, Gesammelte Schriften 10.2, Ffm

Adomo, Th.W. (1980): Minima Moralia, Gesammelte Schriften 4, Ffm

Adorno, Th.W. (1993): Einleitung in die Soziologie (1968), Nachgelassene Schriften, Abt. IV, Bd. 15. Ffm.

Althusser, L. (1978): Die Krise des Marxismus, Hamburg

Brunkhorst, H. (1987): Der Intellektuelle im Land der Mandarine, Ffm

Buchstein, H./Schmalz-Bruns, R. (1992): Gerechtigkeit als Demokratie - Zur politischen Philosophie von Michael Walzer, in: PVS, H. 3, 1992

Demirovic, A. (1992): Führung und Rekrutierung. Die Geburt des Intellektuellen und die Organisation der Kultur, in: Prigge, W. (Hg.): Städtische Intellektuelle. Urbane Milieus im 20. Jahrhundert, Ffm

Demirovic, A. (1993): Politische Führung und Geschlechterverhältnis, Institut für Sozialforschung: Mitteilungen, H. 2, Februar 1993

Foucault, M. (1978a): Dispositive der Macht, Berlin

Foucault, M. (1978b): Der sogenannte Linksintellektuelle, alternative, H. 119, April 1978

Foucault, M. (1990): Was ist Aufklärung?, in: Erdmann, E./Forst,R./Honneth, A. (Hg.): Ethos der Moderne. Foucaults Kritik der Aufklärung, Ffm

Foucault, M. (1992): Was ist Kritik?, Berlin

Horkheimer, M. (1988): Traditionelle und kritische Theorie, Gesammelte Schriften 4, Ffm

Koselleck, R. (1979): Kritik und Krise, Ffm

Lepenies, W. (1992): Das Ende der Utopie und die Rückkehr der Melancholie, in: Meyer (Hg.) 1992

Luhmann, N. (1992): Gibt es ein »System« der Intelligenz?, in: Meyer (Hg.) 1992)

Lyotard, J.-F. (1985): Grabinal der Intellektuellen, Graz

Macho, Th. (1992): Geistesgegenwart. Notizen zur Lage der Intellektuellen, in: Meyer (Hg.) 1992

Meier, C. (1992): Nicht Zerstörung, aber neue Herausforderung der Vernunft, in: Meyer (Hg.) 1992

Meyer, M. (Hg.) (1992): Intellektuellendämnerung? Beiträge zur neuesten Zeit des Geistes, München

Pohrt, W. (1993): Kommunismus oder Barbarei, konkret, H. 2, Februar 1993

Ritter, H. (1992): Der Verdacht, in: Meyer (Hg.) 1992

Ritter, H. (1993): Einkehr zur Weltfremdheit. Hat die Linke noch eine Zukunft?, in: What's Left? Prognosen zur Linken, Berlin

Stephan, C. (1993): Totgesagte leben länger. Die Linke ist so unberechenbar, aber auch so unentbehrlich wie je, in: What's Left? Prognosen zur Linken, Berlin

Walzer, M. (1990): Kritik und Gemeinsinn, Berlin

Walzer, M. (1991): Zweifel und Einmischung, Frankfurt am Main

Walzer, M. (1992a): Chantal Mouffe in Gespräch mit Michael Walzer, Prokla, Heft 87.

Walzer, M. (1992b): Zivile Gesellschaft und amerikanische Demokratie, Berlin

Walzer, M. (1993): Die kommunitaristische Kritik am Liberalismus, in: Honneth, A. (Hg.): Kommunitarismus, Ffm. 